\title{
Immunogénicité et faisabilité de la vaccination intradermique contre la rage au Québec
}

\author{
Bui, $\mathrm{YG}^{1,2,3^{*}}$, Sow $\mathrm{M}^{3}$, Cambron-Goulet $\mathrm{E}^{2}$, Levac $\mathrm{E}^{2}$, Milord $\mathrm{F}^{1,2,3}$ \\ ${ }^{1}$ Institut national de santé publique du Québec, Montréal Québec \\ ${ }^{2}$ Direction de santé publique de la Montérégie, (Québec) \\ ${ }^{3}$ Université de Sherbrooke, Sherbrooke (Québec) \\ *Correspondance : yen.bui.agence16@ssss.gouv.qc.ca
}

\section{Résumé}

Objectifs : La vaccination en préexposition contre la rage est recommandée aux personnes exposées au virus dans le cadre du travail ainsi qu'à certains voyageurs. Avec un coût d'au moins $150 \$$ par dose par voie intramusculaire (IM), cette recommandation est peu suivie. Au Canada, le Comité consultatif national de l'immunisation et le Comité d'immunisation du Québec permettent l'usage de la voie intradermique (ID) qui est une alternative plus économique en utilisant $1 / 10$ de la dose IM, pourvu que certaines conditions soient respectées. Cette étude a pour but d'évaluer la faisabilité et l'immunogénicité de la vaccination en préexposition par voie ID.

Méthodologie : Des étudiants et employés d'une faculté de médecine vétérinaire ont reçu 3 doses de vaccin inactivé sur cellules diploïdes humaines ImovaxRage ${ }^{\mathrm{TM}}$ (sanofipasteur) aux jours 0,7 et 21 ou 28. Une dose de rappel par voie IM ou ID était administrée après 2 ans lorsqu'indiquée.

Résultats : Parmi les 159 participants qui ont reçu 3 doses, 139 ont fait leur sérologie durant l'année suivant la vaccination et tous ont atteint un titre d'anticorps protecteur. Le niveau d'anticorps est plus élevé lorsqu'il est mesuré en-dedans de 5 semaines après la $3^{\mathrm{e}}$ dose. Lors de la sérologie de contrôle 2 ans plus tard, $65 \%$ des participants présentait un dosage $<0,5 \mathrm{UI} / \mathrm{ml}$. Sur les $22 / 30$ qui ont choisi un rappel par voie ID, $100 \%$ ont répondu et les titres moyens d'anticorps ont été multipliés par 11, témoignant d'une forte réponse anamnestique.

Conclusion : La vaccination antirabique par la voie ID est immunogène, économique et pourrait également être envisagée pour la dose de rappel. Les anticorps protecteurs déclinent rapidement après une primovaccination par voie ID, donc il semblerait prudent d'effectuer une sérologie de contrôle 1 an après aux personnes à risque élevé d'exposition occulte dans leur travail. Une alternative serait d'administrer une dose de rappel par voie ID d'emblée à ces personnes 1 an après la primovaccination, ce qui permettrait de simplifier le suivi initial et de réduire les coûts associés (relance, prise de sang, sérologies, etc.). La persistance des anticorps protecteurs après cette dose de rappel devrait être évaluée afin de déterminer la nécessité et le délai entre les sérologies subséquentes.

\section{Introduction}

La rage est une infection causée par un Rhabdovirus du genre Lyssavirus. Elle est transmise par contact avec la salive d'un mammifère infecté, le plus souvent lors d'une morsure. II n'existe aucun moyen de diagnostiquer la maladie avant le début de la phase clinique (1). Le virus cause une encéphalomyélite aigüe et progressive qui est toujours fatale une fois les symptômes apparus, hormis de très rares cas de personnes ayant survécu (2).

Dans le monde, on rapporte plus de 50000 décès liés à la rage chaque année. La majorité des cas est due à une morsure de chien et survient en Asie, en Afrique et en Amérique du Sud. L'Inde compte à elle seule 20000 décès associés chaque année (1). Les voyageurs qui visitent les régions où la rage est fortement endémique sont à risque, particulièrement s'ils circulent en zones rurales (3). Dans plusieurs pays d'Europe, la majorité des cas humains rapportés sont importés et surviennent chez des voyageurs (4).

Au Canada, on a dénombré seulement 24 cas de rage humaine entre 1924 et 2009 (5) avec les trois derniers cas attribuables à des chauves-souris. D'ailleurs, la majorité des cas de rage surviennent à la suite d'un contact avec 
une chauve-souris infectée $(6,7)$, même si le réservoir principal de la rage terrestre est le renard roux. Bien que les cas de rage animale soient en déclin au Canada depuis 2000, passant de 670 à 141 en 2012 (8), le réservoir animal de la maladie demeure vaste (5). Les personnes en contact avec des animaux dans le cadre de leur travail, par exemple les vétérinaires, sont plus à risque d'être exposés au virus de la rage.

En plus de la voie d'administration intramusculaire (IM), l'Organisation Mondiale de la Santé (OMS) permet l'usage intradermique (ID) des vaccins antirabiques préparés en culture cellulaire ou sur des œufs embryonnés, en autant qu'ils contiennent $2,5 \mathrm{UI}$ par dose IM $(6,9)$. Cette mesure sert avant tout à favoriser dans les pays en voie de développement, l'utilisation de ces vaccins en postexposition, lesquels ont un coût plus élevé mais sont nettement plus efficaces et entraînent beaucoup moins d'effets indésirables graves que les vaccins préparés à partir de tissus nerveux animaux $(1,6)$. Un protocole de vaccination en post-exposition par voie ID a été introduit pour la première fois en Thaïlande en 1984 et par la suite implanté avec succès entre autres en Inde, aux Philippines, au Sri Lanka et en Thaïlande $(10,11)$.

Plusieurs organismes scientifiques entérinent également l'utilisation de la voie ID en préexposition contre la rage, dont l'OMS et le Comité consultatif national de l'immunisation au Canada (CCNI). Cette pratique permet de réduire les coûts de la vaccination en préexposition qui n'est pas soutenue financièrement par le système public canadien, chaque dose de vaccin coûtant de 150 à 180\$. Elle permet également de simplifier la conduite en postexposition, éliminant la nécessité des immunoglobulines contre la rage, et réduisant le nombre de doses de vaccins requis de 4 à 2 (12).

Au Québec, la vaccination est encadrée légalement par le Protocole d'immunisation du Québec (PIQ), ouvrage produit par le ministère de la Santé et des Services sociaux et entériné par un comité scientifique aviseur, le Comité d'immunisation du Québec. Selon le PIQ, la vaccination contre la rage en préexposition peut ainsi se faire avec $0,1 \mathrm{ml}$ par voie ID ou avec $1 \mathrm{ml}$ par la voie IM, en utilisant l'un ou l'autre des vaccins homologués au Canada, RabAvert ${ }^{\mathrm{TM}}$ (Novartis) préparé sur des cellules d'embryons de poulet, et ImovaxRage ${ }^{\mathrm{TM}}$ (sanofipasteur) préparé sur des cellules diploïdes humaines, les deux reconnus comme immunogènes et considérés comme interchangeables (13). La voie ID nécessite une bonne maîtrise de la technique d'injection pour éviter l'administration en sous-cutanée et les règles de conservation et d'asepsie doivent être respectées (6). De plus, une sérologie après la vaccination par voie ID est requise selon le Guide canadien d'immunisation et le PIQ pour documenter la réponse immunitaire car aucune formulation ID n'est homologuée au Canada. Enfin, pour réaliser des économies, il est nécessaire de regrouper plusieurs personnes lors d'une même séance de vaccination. Pour toutes ces raisons, cette voie d'administration est peu utilisée.

Plusieurs études ailleurs dans le monde ont démontré l'immunogénicité de la vaccination ID en préexposition (1417). De plus, la hausse des taux d'anticorps provoquée par l'administration d'une dose de rappel IM ou ID chez les personnes préalablement vaccinées par voie ID est aussi adéquate que chez celles vaccinées préalablement par voie IM, témoignant d'une bonne réponse anamnestique $(18,19)$.

Notre étude vise à démontrer l'immunogénicité et la faisabilité de la vaccination ID contre la rage dans le contexte canadien. Cette démonstration permettrait de systématiser l'offre vaccinale auprès des groupes à risque et ainsi d'améliorer l'accès au vaccin antirabique.

\section{Méthodologie}

Dans le but d'offrir la vaccination contre la rage en préexposition à moindre coût, la Faculté de médecine vétérinaire de l'Université de Montréal située à St-Hyacinthe, Québec, Canada a une entente de service avec le Centre de santé et de services sociaux (CSSS) Richelieu-Yamaska pour la vaccination par voie ID des étudiants et employés de cette Faculté. Les participants ont été recrutés par lettre et sur une base volontaire pour se prémunir de la vaccination antirabique par voie ID à l'automne 2006. Les femmes enceintes, les personnes âgées de moins de 18 ans, les personnes déjà vaccinées, celles ayant déjà présenté une réaction allergique grave à une dose antérieure du vaccin ou à l'une de ses composantes ainsi que les personnes prenant de la chloroquine ou devant débuter la prise de ce médicament moins d'un mois après la vaccination étaient exclues.

Le vaccin ImovaxRage ${ }^{\mathrm{TM}}$ (sanofipasteur) a été utilisé. II s'agit d'un vaccin lyophilisé, obtenu sur cellules diploïdes humaines (VCDH), contenant 2,5 IU/ml (13). Étant donné l'absence au Canada de formulations prévues pour 
l'injection ID, les séances de vaccination ont regroupé un nombre suffisant de participants pour permettre le fractionnement de doses de 0,1 $\mathrm{ml}$ à partir de fioles unidoses de $1 \mathrm{ml}$. Conformément au PIQ, les vaccins ont été conservés entre 2 et $8^{\circ} \mathrm{C}$ et aucune dose n'a été administrée plus de 6 heures après la reconstitution du produit. Les règles d'asepsie ont été rigoureusement respectées.

Un consentement écrit était obtenu au moment de la vaccination. Un consentement écrit a aussi été obtenu pour la transmission à l'équipe de recherche des informations sur les dates de vaccination, les données démographiques, les suivis effectués et les résultats des sérologies. Des infirmières expérimentées et maîtrisant bien la technique d'injection ID ont procédé à la vaccination à la Faculté de médecine vétérinaire entre le 11 septembre et le 6 novembre 2006. Trois doses ID de 0,1 $\mathrm{ml}$ ont été administrées dans la région du deltoïde aux jours $0,7,21$ ou 28. Un système de rappel par téléphone et par courrier a été mis en place. Un formulaire permettait de recueillir des informations démographiques sur les participants ainsi que les manifestations cliniques graves ou inhabituelles survenant après la vaccination.

Une première sérologie a été prescrite de 2 à 4 semaines après la troisième dose du vaccin, ce qui est le délai recommandé par le PIQ (13), afin de s'assurer de l'immunité conférée. Pour les personnes dont le dosage d'anticorps était inférieur à $0,5 \mathrm{UI} / \mathrm{ml}$, une dose supplémentaire du vaccin par voie IM ou ID était offerte. Une $2^{\mathrm{e}}$ sérologie était recommandée à ces personnes afin de s'assurer d'une réponse immunitaire adéquate.

Pour les personnes ayant eu une réponse immunitaire adéquate, une sérologie de contrôle a été réalisée deux ans après la complétion de la série vaccinale afin de vérifier la persistance des anticorps. À cette étape, les sujets pouvaient choisir de recevoir une dose de rappel administrée par voie ID ou IM si leur dosage d'anticorps était inférieur à $0,5 \mathrm{Ul} / \mathrm{ml}$. Tous les dosages d'anticorps ont été effectués à Toronto, au Laboratoire national canadien de référence contre la rage, par la technique de réduction des foyers de fluorescence (RFFIT).

Les caractéristiques démographiques des participants suivis ont été comparées à celles des participants perdus de vue par le test du Chi-deux ou le test $\mathrm{T}$. La corrélation entre le délai de la première sérologie et le dosage d'anticorps a été mesurée par le coefficient de Pearson. Un seuil de signification de 0,05 a été retenu pour les analyses et des intervalles de confiance (IC) à 95\% ont été calculés lorsque pertinent. Le protocole de recherche de cette étude a été approuvé par le comité d'éthique de la recherche du CSSS Richelieu-Yamaska.

\section{Résultats}

Un total de 159 étudiants et employés de la Faculté de médecine vétérinaire ont reçu les trois doses prévues de vaccin (Figure 1) et ont consenti à ce que leurs informations soient transmises à l'équipe de recherche. Les sujets étaient âgés de 18 à 59 ans (moyenne de 23 ans et médiane de 22 ans) et 84\% était des femmes. Aucune manifestation clinique grave ou inhabituelle après la vaccination n'a été rapportée. 
Figure 1: Diagramme de suivi des participants

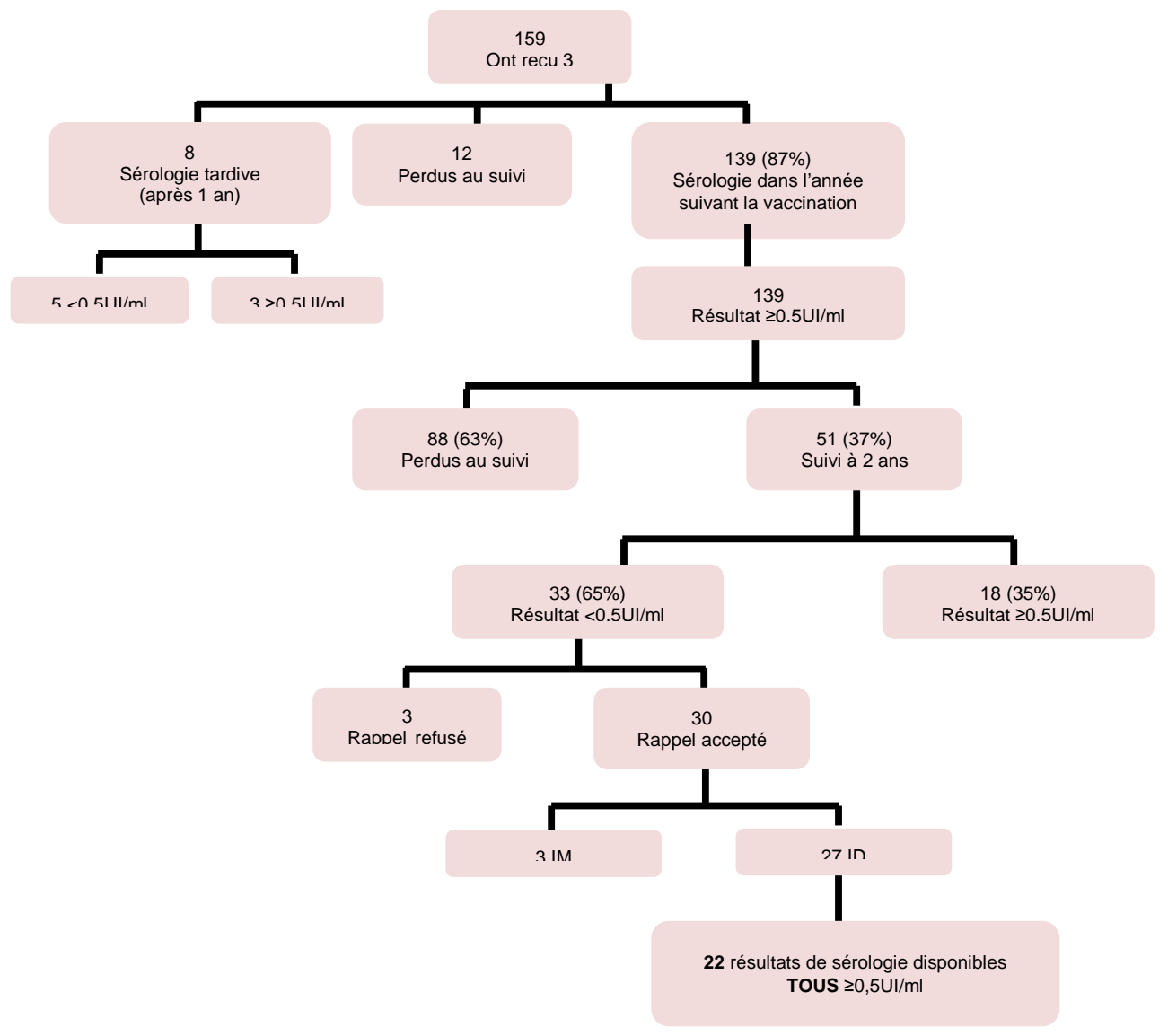

Le résultat de la première sérologie était disponible pour 147 personnes (Figure 2), dont 139 ayant eu leur sérologie dans l'année suivant la complétion de la série vaccinale, avec un délai variant de 2 à 11 semaines entre la $3^{\mathrm{e}}$ dose de vaccin et la sérologie. Les personnes ayant fait leur sérologie 2 semaines après avaient en moyenne une concentration d'anticorps de $6,1 \mathrm{Ul} / \mathrm{ml}$, tandis que cette concentration était en moyenne de 2,6 $\mathrm{Ul} / \mathrm{ml}$ si la sérologie était faite 5 semaines après la $3^{\mathrm{e}}$ dose. Il y a une corrélation négative entre le délai entre la $3^{\mathrm{e}}$ dose et la concentration d'anticorps atteinte: $r=-0,31 ; p<0,05$. Des huit personnes ayant fait leur sérologie 1 an ou plus après la vaccination (le délai pouvait aller jusqu'à 161 semaines), cinq avaient un résultat <0,5 Ul/ml.

Figure 2: Moyenne géométrique des titres ( $\mathrm{Ul} / \mathrm{ml}$ ) selon le délai depuis la 3e dose ( $\mathrm{N}=147)$

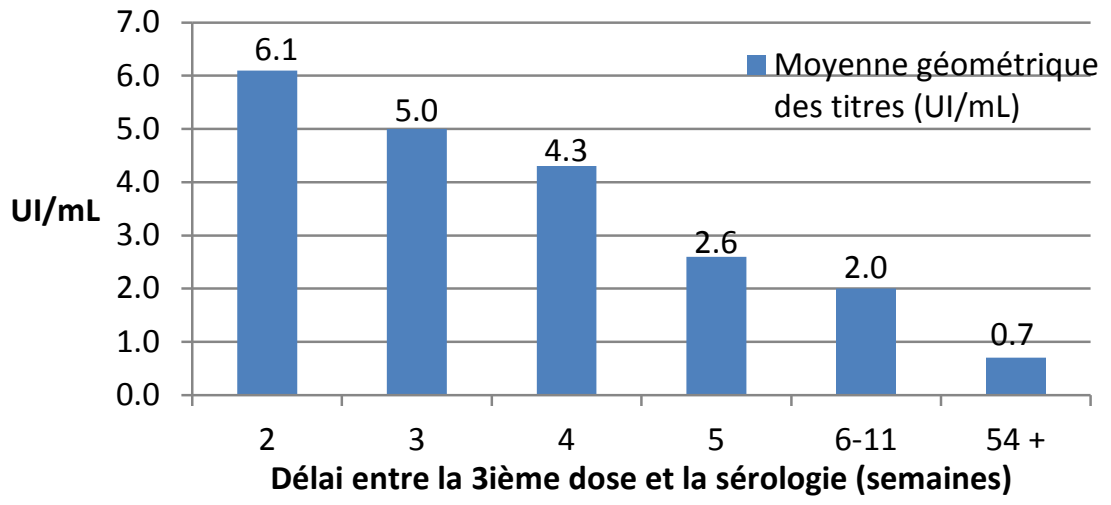


Des 139 participants (87\%) ayant fait leur sérologie dans la première année (de 2 à 11 semaines après la $3^{\mathrm{e}}$ dose), $100 \%$ ont obtenu un taux d'anticorps antirabiques $\geq 0,5 \mathrm{Ul} / \mathrm{ml}$ (Tableau 1). Deux ans après la complétion de la série vaccinale, 51 participants se sont présentés pour une sérologie de contrôle. Bien qu'on observe une diminution généralisée des anticorps entre la première et la deuxième sérologie, 35\% (18/51) avaient toujours un niveau d'anticorps antirabiques protecteur. D'après les résultats disponibles pour 22 participants qui ont accepté le rappel par voie ID, $100 \%$ ont obtenu un taux d'anticorps antirabiques $\geq 0,5 \mathrm{UI} / \mathrm{ml}$ et on note une forte augmentation dans les concentrations d'anticorps (Tableau 1). II n'y a pas de différence entre les participants ayant fait l'objet d'un suivi et ceux perdus de vue concernant l'âge moyen (22 ans contre 24 ans, $p=0,44)$ et la proportion de femmes ( $82 \%$ contre $85 \%, p=0,63)$.

Tableau 1: Titre d'anticorps (UI/ml) après la 3e dose, lors de la sérologie de contrôle deux ans après, et après la dose de rappel

\begin{tabular}{|l|c|c|c|}
\hline \multicolumn{1}{|c|}{ Variables } & $\begin{array}{c}\text { Première sérologie (2- } \\
\mathbf{1 1} \text { semaines après la } \\
\mathbf{3}^{\mathrm{e}} \text { dose) }\end{array}$ & $\begin{array}{c}\text { Sérologie de suivi } \\
\text { après 2 ans }\end{array}$ & $\begin{array}{c}\text { Sérologie après la } \\
\text { dose de rappel }\end{array}$ \\
\hline Nombre de cas & 139 & 51 & 22 \\
\hline $\begin{array}{l}\text { Concentration moyenne } \\
\text { des titres d'anticorps } \\
(\text { Ul/ml) } \\
{[\text { IC à 95\%] }}\end{array}$ & $5,3[4,9-5,7]$ & $0,74[0.4-1]^{1}$ & $4,8[4,1-5,5]$ \\
\hline Étendue & $0,6-10,0$ & $0,1-5,7$ & $1,5-7,4$ \\
\hline $\begin{array}{l}\text { Titre protecteur } \\
\text { d'anticorps }\end{array}$ & $100 \%(139 / 139)$ & $35 \%(18 / 51)$ & $100 \%(22 / 22)$ \\
\hline
\end{tabular}

${ }^{1}$ Excluant un participant qui a montré une augmentation des concentrations d'anticorps entre la première et la deuxième sérologie

\section{Conclusion}

À notre connaissance, il s'agit de la première étude soumise pour publication sur la vaccination intradermique contre la rage en préexposition au Canada. Un total de 159 participants ont reçu une série vaccinale de trois doses de 0,1 ml du vaccin ImovaxRage ${ }^{\mathrm{TM}}$ (sanofipasteur) par voie ID. Aucun effet indésirable grave n'a été noté. II semblerait que les effets indésirables observés avec la voie ID dans le passé ont diminué grâce à une technique d'administration mieux maîtrisée et à l'efficacité et la sécurité des nouvelles générations de vaccins antirabiques $(20,21)$. Tous les participants qui se sont soumis à une sérologie durant l'année suivant la troisième dose avaient des niveaux d'anticorps antirabiques protecteurs. Ces résultats confirment que l'administration par voie ID du vaccin antirabique est sécuritaire et immunogène chez les personnes en bonne santé.

Nous observons une diminution rapide des titres d'anticorps si la sérologie est faite plus de 5 semaines après la primovaccination. Notre étude ne comporte pas de groupe contrôle par voie IM afin de mesurer si la voie d'administration a une influence sur la cinétique de la réponse immunitaire. Certaines études ont rapporté que les titres atteints après une primovaccination et une dose de rappel étaient plus élevés par voie IM que par voie ID $(18,22,23)$.Toutefois, l'atteinte d'un seuil d'anticorps protecteur serait aussi adéquate par voie ID que par voie IM $(6,21)$. De plus, on sait que les vaccins antirabiques sur culture cellulaire procurent une excellente mémoire immunitaire et plusieurs études ont mis en évidence une réponse anamnestique à la suite d'une dose de rappel chez des personnes préalablement vaccinées par voie ID $(19,24,25,26)$.

Nos résultats démontrent que deux années après la primovaccination, les anticorps antirabiques persistent à des niveaux variables, en l'occurrence $35 \%$ des sujets avaient encore un titre protecteur. Après l'administration du rappel, $100 \%$ des participants pour lesquels un résultat de sérologie était disponible ont développé une réponse anamnestique avec des titres d'anticorps moyens multipliés par 11. Les participants à cette étude étaient tous âgés de 18 ans et plus et pour une grande majorité des femmes, reflétant la démographie des étudiants dans cette faculté de médecine vétérinaire. Aucune différence significative entre les groupes d'âge ou le sexe n'a été relevée dans cette étude comme dans la littérature (26). Les caractéristiques démographiques des participants perdus de vue étaient similaires à celles des participants ayant complété l'étude. 
Plusieurs études ont montré que la réponse anamnestique à une dose de rappel peut être induite des années après la primovaccination, même chez des personnes dont les titres d'anticorps ont baissé en bas du seuil considéré comme protecteur de $\geq 0,5 \mathrm{UI} / \mathrm{ml}(26,27)$. Malerczyk et al rapportaient une réponse anamnestique à la dose de rappel chez des personnes vaccinés 15 ans auparavant (25) et d'autres études parlent d'un effet anamnestique en réponse à une dose de rappel jusqu'à 21 ans après une primovaccination (6).

Au cours des dernières années, on observe que la rage est en émergence dans le Sud du Québec avec la migration d'une nouvelle souche de la rage du raton-laveur en provenance des États-Unis $(27,28)$. Les participants à cette étude sont des étudiants en médecine vétérinaire qui oeuvrent dans une zone enzootique. Or, nos résultats montrent que les titres d'anticorps baissent rapidement après la $3^{\mathrm{e}}$ dose et qu'un an après la primovaccination, un certain nombre de vaccinés ont déjà un titre inférieur à $0,5 \mathrm{Ul} / \mathrm{ml}$. Une revue systématique réalisée en Angleterre rapportait que jusqu'à $13 \%$ des vaccinés voyaient leur taux d'anticorps baisser à $<0,5$ $\mathrm{UI} / \mathrm{ml}$ une année après la primovaccination par voie IM (29). Comme les titres d'anticorps peuvent être moins élevés avec la voie ID $(25,26)$, on s'attend à ce que la baisse des anticorps soit plus rapide après cette voie d'administration. Nos résultats vont dans le même sens que cette étude et suggèrent que, pour les groupes à risque d'exposition occulte, le délai pour la première sérologie de contrôle et l'administration de la dose de rappel devrait être devancée à un an au lieu de deux. Cette recommandation permettrait de s'assurer que le titre d'anticorps de ces personnes ne descende pas en dessous du seuil protecteur.

Actuellement, le délai pour l'obtention du résultat de la sérologie est d'au moins 6 à 8 semaines. De plus, le suivi des patients et les relances pour les prises de sang mobilisent beaucoup de ressources et le nombre de patients perdus au suivi demeure problématique (63\% dans notre étude). Plus d'études sont nécessaires afin de documenter la persistance des anticorps protecteurs après une primovaccination par voie ID et après une dose de rappel par voie ID, mais nous pensons qu'un rappel administré à un an par voie ID pourrait conférer une protection assez longue(5) pour permettre d'espacer le délai entre les sérologies de contrôle subséquentes.

En effet, on sait que les vaccins sur cultures cellulaires sont très immunogènes et que les titres d'anticorps protecteurs après une dose de rappel peuvent durer jusqu'à cinq ans dans $96,2 \%$ des cas $(5,6)$. Une étude a démontré qu'une dose de rappel administrée 1 an après la série vaccinale primaire pouvait induire un titre d'anticorps protecteurs persistant jusqu'à 10 ans (30). Selon cette étude, les sujets ayant atteint un titre $\geq 30$ $\mathrm{UI} / \mathrm{ml}$ pourraient recevoir un rappel à tous les 10 ans, et ceux avec un titre $<30 \mathrm{UI} / \mathrm{ml}$ un rappel aux 3 ans. Cette conduite éliminerait la nécessité de faire des sérologies aux 2 ans.

Cinq participants ayant fait leur sérologie 1 an ou plus après la $3^{\mathrm{e}}$ dose ont obtenu un résultat en-dessous du seuil protecteur. Nos données ne nous permettent pas de déterminer s'il s'agit d'un échec primaire à la vaccination ou d'une baisse graduelle attendue des concentrations d'anticorps avec le temps. Étant donné l'excellente réponse immunitaire chez les 139 participants ayant fait leur sérologie durant la $1^{\text {re }}$ année, nous supposons que si la sérologie avait été faite plus tôt après la primovaccination, ces cinq personnes auraient possiblement atteint un niveau d'anticorps adéquat.

Nous avons pu directement constater l'avantage en terme économique de cette voie d'injection lors des séances de vaccination. Une fiole de vaccin antirabique de $1 \mathrm{ml}$ suffisait pour vacciner de 7 à 8 personnes par voie ID. Le CSSS a établi le coût par dose de vaccin ID à $60 \$$, soit 3 fois moins cher que pour une dose IM. Pour calculer les économies nettes, il faudrait inclure dans les coûts le temps infirmier requis pour la technique ID, les coûts de la sérologie, les relances et les suivis des résultats. Nous observons par ailleurs que $90 \%$ des participants dont le niveau d'anticorps antirabique étaient de $<0.5 \mathrm{IU} / \mathrm{ml}$ après deux ans ont opté pour la voie d'administration ID pour la dose de rappel malgré la nécessité de répéter une autre sérologie pour documenter la réponse.

Par ailleurs, nous savons que le vaccin contre la rage est peu accepté par les voyageurs. Une étude réalisée à l'aéroport de Bangkok auprès de 7681 voyageurs révélait que seulement $12 \%$ avait complété leur série vaccinale, $15 \%$ ayant reçu une ou deux doses et $73 \%$ n'étant pas vaccinés (31). Le risque de morsure lors de voyage dans des zones endémiques est difficile à évaluer. Une étude menée auprès de 1882 touristes visitant la Thaïlande pour une durée moyenne de dix-sept jours estimait à 13 le nombre de morsures sur 1000 touristes (32). La réduction des coûts est essentielle afin d'améliorer l'accès au vaccin pour les groupes présentant un risque professionnel comme les participants à cette étude ou les voyageurs en zones d'enzootie. Un autre avantage de vacciner les voyageurs en préexposition serait d'éliminer la nécessité de recourir aux immunoglobulines contre la 
rage en cas de morsures subies à l'étranger, qui sont très peu disponibles dans la plupart des pays en voie de développement (6), et de réduire le nombre de doses de vaccin requises en postexposition. De plus, les voyageurs, n'étant pas considérés comme à risque d'exposition occulte au virus de la rage, n'ont pas à recevoir de dose de rappel ni à se soumettre à des sérologies de contrôle répétées.

D'autres calendriers vaccinaux par voie ID en préexposition ont été étudiés ailleurs dans le monde, tels que l'administration de deux injections ID simultanées (une dans chaque deltoïde) aux jours $0,7,21$ ou 28, aux jours $0,3,7$ ou seulement au jour 0 (19). Indépendamment du calendrier utilisé, une forte réponse anamnestique est observée 1 an plus tard à la suite de l'administration de deux doses de rappel. Bien qu'il s'agisse de pratiques empiriques, elles nous semblent intéressantes pour circonvenir au manque de temps pour effectuer la sérologie dans le cas de voyageurs à haut risque avec un départ prévu dans un court délai.

Un décès dû à la rage à la suite d'une morsure de chien survenue au Kenya chez une coopérante américaine a été rapporté en 1983 (33). Le cas avait été vacciné huit mois auparavant au Kenya par voie ID avec une série de 3 doses d'un vaccin sur cellules diploïdes humaines, mais n'avait pas consulté à la suite de la morsure afin de recevoir la vaccination en postexposition. Une sérologie faite lors de l'apparition de ses symptômes a révélé un titre d'anticorps inadéquat. Par la même occasion, des sérologies effectuées chez les autres membres du Corps des volontaires de la paix également vaccinés par voie ID ont révélé que 9 sujets sur 11, présentaient des niveaux d'anticorps non protecteurs. Bien que la réponse après primovaccination ne semblait pas adéquate, l'enquête considère que si deux doses avait été administrées en post-exposition tel que recommandé, le décès aurait pu être évité. Ceci rappelle l'importance de la sérologie de contrôle à la suite d'une primovaccination par voie ID pour s'assurer que les taux d'anticorps soient $\geq 0,5 \mathrm{Ul} / \mathrm{ml}$. Cet incident, ainsi qu'une série de trois lots de vaccins homologués pour la voie ID avec une concentration d'antigènes insuffisante, ont mené au retrait des vaccins ID du marché américain en 2001, même si l'immunogénicité de la voie ID n'est pas remise en cause (3).

La vaccination par la voie ID continue d'être offerte à la Faculté de médecine vétérinaire de l'Université de Montréal. Entre 2007 et 2013, près de 1000 personnes ont été vaccinées et seulement deux résultats $<0,5 \mathrm{Ul} / \mathrm{ml}$ ( 0,36 et $0,47 \mathrm{Ul} / \mathrm{ml}$ respectivement) ont été observés sur des sérologies faites 2 à 4 semaines après la $3^{\mathrm{e}}$ dose (Jocelyne Angers, communication personnelle, juillet 2014). Cette voie est toujours bien tolérée, et lors des séances les vaccins RabAvert ${ }^{\mathrm{TM}}$ et ImovaxRage ${ }^{\mathrm{TM}}$ sont utilisés de façon interchangeable facilitant ainsi la gestion des stocks de vaccin.

Nos résultats démontrent que l'injection de 1/10 de la dose IM par voie ID est une alternative immunogène, économique et faisable dans des milieux où le regroupement de la clientèle est possible, avec un personnel qualifié et maîtrisant bien la technique, Iorsqu'on dispose d'assez de temps pour effectuer la sérologie postvaccinale. Le contexte de notre étude (faculté de médecine vétérinaire) regroupait toutes ces conditions. Comme les titres d'anticorps chutent rapidement dans la $1^{\text {re }}$ année, l'administration d'une dose de rappel un an après la primovaccination aux personnes à risque élevé d'exposition occulte à la rage sans faire de sérologie au préalable serait une approche prudente selon nous. Un suivi sérologique pour documenter la persistance des anticorps devrait être fait par la suite afin de déterminer la nécessité et le moment le plus opportun pour d'autres doses de rappel.

La disponibilité d'une formulation homologuée pour la voie ID au Canada et le raccourcissement du délai pour le résultat de sérologie atténueraient les problèmes logistiques rencontrés dans notre étude.

\section{Remerciements}

Nous remercions les étudiants et les employés de la Faculté de médecine vétérinaire de l'Université de Montréal pour leur contribution à cette étude. Un grand merci à toute l'équipe du CSSS Richelieu-Yamaska et en particulier à Francine Lord et Danielle Bisson pour les aspects logistiques. Nous remercions également Mme Anne-Marie Clouâtre, Dre Lina Perron et Dre Louise Lambert de la Direction de santé publique de la Montérégie pour la révision critique du projet et Nathalie Bernier et Marianne Picard-Masson pour certaines analyses.

\section{Conflits d'intérêt}

Les auteurs n'ont signalé aucun conflit d'intérêt. 


\section{Références}

(1) Organisation mondiale de la santé. Rabies vaccines. Wkly Epidemiol Rec 2010;85(32):309-20.

(2) Willoughby RE, Tieves KS, Hoffman GM, Ghanayem NS, Amlie-Lefond CM, Schwabe MJ, Chusid MJ, Rupprecht CE. Survival after treatment of rabies with induction of coma. N Engl J Med 2005;352(24):2508-14.

(3) Rupprecht CE, Shlim DR. Rabies. In: Centers for Disease Control and Prevention (CDC). CDC Health Information for International Travel 2014. New York: Oxford University Press, 2014:270-6.

(4) Comité consultatif de la médecine tropicale et des voyages. Déclaration relative aux voyageurs et au vaccin contre la rage. RMTC 2002;28. http://www.collectionscanada.gc.ca/webarchives/20071116052847/http://www.phacaspc.gc.ca/publicat/ccdr-rmtc/02vol28/28sup/dcc4.html_Consulté le 21 juillet 2014. Available in English : http://www.collectionscanada.gc.ca/webarchives/20071116023105/http://www.phac-aspc.gc.ca/publicat/ccdrrmtc/02vol28/28sup/acs4.html

(5) Agence de santé publique du Canada. Guide canadien d'immunisation. Partie 4 - Vaccins inactivés, Vaccins contre la rage. http://www.phac-aspc.gc.ca/publicat/cig-gci/p04-rabi-rage-fra.php\#voyageurs Consulté le 21 juillet 2014.

(6) Organisation mondiale de la santé. WHO Expert Consultation on rabies. World Health Organ Tech Rep Ser 2005;931:1-88. http://whqlibdoc.who.int/trs/WHO_TRS_931_eng.pdf Consulté le 21 juillet 2014.

(7) De Serres G, Dallaire F, Côte M, Skowronski DM. Bat rabies in the United States and Canada from 1950 through 2007: Human Cases with and without bat contact. Clin Inf Dis 2008;46:1329-37.

(8) Agence canadienne d'inspection des aliments. Cas de rage positifs au Canada - Rage chez les animaux. http://epe.lac-bac.gc.ca/100/206/301/cfia-acia/2011-09-

21/www.inspection.gc.ca/francais/anima/disemala/rabrag/statsf.shtml

Consulté le 21 juillet 2014.

(9) World Health Organization. Updated WHO position paper on rabies vaccines. Geneva, 2010. En ligne http://www.who.int/immunization/Rabies_slides_Aug_2010.pdf

Consulté le 21 juillet 2014.

(10) Chulasugandha P, Khawplod P, Havanond P, Wilde H. Cost comparison of rabies pre-exposure vaccination with post-exposure treatment in Thai children. Vaccine 2006;24(9):1478-82.

(11) Quiambao BP, Dimaano EM, Ambas C, Davis R, Banzhoff A, Malerczyk C. Reducing the cost of post-exposure rabies prophylaxis: efficacy of $0.1 \mathrm{ml}$ PCEC rabies vaccine administered intradermally using the Thai Red Cross postexposure regimen in patients severely exposed to laboratory-confirmed rabid animals. Vaccine 2005;23(14):1709-14.

(12) Comité national consultatif de l'immunisation. Mise à jour sur les vaccins antirabiques. RMTC 2005;31.

(13) Ministère de la santé et des services sociaux du Québec. Protocole d'immunisation du Québec - $6^{\mathrm{e}}$ édition. Québec : Gouvernement du Québec, 2013.

(14) Lau C, Sisson J. The effectiveness of intradermal pre-exposure rabies vaccination in an Australian travel medicine clinic. J Travel Med 2002;9(6):285-8.

(15) Wilde H, Briggs DJ, Meslin FX, Hemachudha T, Sitprija V. Rabies update for travel medicine advisors. Clin Infect Dis 2003;37(1):96-100.

(16) Ranney M, Partridge R, Jay GD, Rozzoli DE, Pandey P. Rabies antibody seroprotection rates among travelers in Nepal: "rabies seroprotection in travelers". J Travel Med 2006;13(6):329-33.

(17) Bui YG, Milord F, Levac E, Lord F. Faisabilité de la vaccination intradermique contre la rage au Québec. Communication par affiche présentée aux $10^{\mathrm{e}}$ Journées annuelles de santé publique. Montréal (QC), 2006.

(18) Jijaroensup W, Limusanno S, Kwawplod P, Serikul K, Chomchay P, Kaewchomphoo W, Tantawichien T, Wilde H. Immunogenicity of rabies postexposure booster injections in subjects who had previously received intradermal preexposure vaccination. J Travel Med 1999;6:234-37.

(19) Khawplod p, Wilde H, Benjavongkulchai M, Srianroon C, Chomchey P. Immunogenicity study of abbreviated rabies preexposure vaccination schedules. J Travel Med 2007;14(3):173-6.

(20) Briggs DJ, Banzhoff A, Nicolay U, Sirikwin S, Dumavibhat B, Tongswas S, Wasi C. Réponse en anticorps après vaccination antirabique de post-exposition par de petites doses intradermiques de vaccin purifié préparé sur cellules d'embryon de poulet ou sur cellules Vero. Bull World Health Organ 2000;78:693-8.

(21) World Health Organization. WHO position paper on rabies vaccine - Table III: Safety of cell-culture-based rabies vaccines. Geneva, 2010. http://www.who.int/immunization/rabies_grad_safety.pdf Consulté le 21 juillet 2014.

(22) Kositprapa C, Limsuwun K, Wilde H, Jaijaroensup W, Saikasem A, Khawplod A, Kri-aksorn U, Supich C. Immune response to stimulated postexposure rabies booster vaccinations volunteers who received preexposure vaccinations. Clin Inf Dis 1997;25:614-6.

(23) Nicholson KG, Turner GS, Aoki FY. Immunization with a human diploid cell strain of rabies virus vaccine: two-year results. J Infect Dis 1978;137(6):783-8.

(24) Suwansrinon K, Wilde H, Benjavongkulchai U, Lertjarutorn S, Boonchang S, Suttisri R, Khowplod P, Daviratanasilpa S, Sitprija V. Survival of neutralizing antibody in previously rabies vaccinated subject: a prospective study showing long lasting immunity. Vaccine 2006;24(18):3878-80.

(25) Malerczyk C, Briggs DJ, Dreesen DW, Banzhoff A. Duration of immunity: an anamnestic response 14 years after rabies vaccination with purified chick embryo cell rabies vaccine. J Travel Med 2007;14(1):63-4. 
(26) Brown D, Featherstone JJ, Fooks AR, Gettner S, Lloyd E, Schweiger M. Intradermal pre-exposure rabies vaccine elicits long lasting immunity. Vaccine 2008;26:3909-12.

(27) Chang HG, Eidson M, Noonan-Toly C, Trimarchi CV, Rudd R, Wallace BJ, Smith PF, Morse DL. Public health impact of reemergence of rabies, New York. Emerg Infect Dis 2002;8(9):909-13.

(28) Blanton JD, Dyer J, McBrayer J, Rupprecht CE. Rabies surveillance in the United States during 2011. J Am Vet Med Assoc 2012;241(6):712-22.

(29) Morris J, Crowcroft NS. Pre-exposure rabies booster vaccinations: a literature review. Dev Biol (Basel) 2006;125:20515.

(30) Strady A, Lang J, Lienard M, Blondeau C, Jaussaud R, Plotkin SA. Antibody persistence following preexposure regimens of cell-culture rabies vaccines : 10-year follow-up and proposal for a new booster policy. $J$ Inf Dis 1998;177:1290-5.

(31) Piyaphanee W, Kittitrakul C, Lawpoolsri S, Gautret P, Kashino W, Tangkanakul W, Charoenpong P, Ponam T, Sibunruang S, Phumratanaprapin W, Tantawichien T. Risk of potentially rabid animal exposure among foreign travelers in Southeast Asia. PLoS Negl Trop Dis 2012;6(9):e1852. doi: 10.1371/journal.pntd.0001852

(32) Phanuphak P, Ubolyam S. Should travelers in rabies endemic areas receive pre-exposure rabies immunization? Presentation at the International Conference on Travellers' Medicine. Atlanta (GA), 1991.

(33) Bernard KW, Fishbein DB, Miller KD, Parker RA, Waterman S, Summer JW, Reid FL, Johnson BK, Rollins AJ, Oster $\mathrm{CN}$ et al. Pre-exposure rabies immunization with human diploid cell vaccine: decreased antibody responses in persons immunized in developing countries. Am J Trop Med Hyg 1985;34(3):633-47. 